Walter G. Bradley

W. G. Bradley (ه)

DM, FRCP. Professor and Chairman,

Department of Neurology,

University of Miami School of Medicine,

PO Box 016960,

Miami, FL 33101, USA

e-mail: wbradley@med.miami.edu

\title{
Biological markers in amyotrophic lateral sclerosis: help or hindrance?
}

\begin{abstract}
Biological markers play an important role in the development of the understanding of a disease, its diagnosis and treatment. This is particularly true of amyotrophic lateral sclerosis (ALS) at this time. We need better biological markers for the diagnosis, for improved understanding of the underlying pathogenetic mechanisms, and for assistance in new drug development. This review of
\end{abstract}

currently available biological and surrogate markers in ALS discusses novel approaches to the use of such markers, and new drugs for the treatment of ALS.

Key words Amyotrophic lateral sclerosis - Biological markers . Surrogate markers - Therapeutic trials $\cdot$ Etiopathogenesis

\section{Introduction}

This review will consider the role of biological and surrogate markers in the diagnosis of amyotrophic lateral sclerosis (ALS), in measuring disease progression during therapeutic trials in ALS, and in developing potential new therapies for the disease. A biological marker is an indicator of a change associated with the disease, and can be either a marker of the direct cause of the disease or a secondary effect. Currently the primary underlying cause or causes of ALS are unknown, although many biological changes resulting from the disease process have been recognized. The cascade of biochemical changes occurring within the motor system in ALS leads eventually to degeneration of the lower and upper motor neurons (LMNs and UMNs), and it is this degeneration which is responsible for the clinical symptoms and signs of the disease. Ideally, we should use direct evidence of motor neuronal degeneration as the primary biological marker in studies of ALS, but since brain or spinal cord biopsies cannot be obtained we are forced to use secondary or surrogate markers.

The term 'surrogate marker' is frequently used in discussions about biological markers. A surrogate marker is a substitute for another marker, with the implication that the surrogate is not as good as the original. The term usually refers to disease-related epi-phenomena that nevertheless can be used in the diagnosis or measurement of progression of the disease.

\section{Use of biological markers in the diagnosis of patients with symptoms suggestive of ALS}

If there is a known family history of similar disease in a patient presenting with signs and symptoms suggestive of ALS, genetic techniques may be able to establish the diagnosis. These can screen for mutations of known genes such as the SOD-1 gene in familial ALS. If the chromosomal localization but not the actual gene is known, linkage to other genes and haplotype analysis can be used.

The diagnosis of ALS in the early stages can be difficult because there may not be clear evidence of both $\mathrm{UMN}$ and LMN involvement and of multi-level damage. Biological markers can be used to clarify the diagnosis. Evidence of LMN denervation on electromyography can assist in confirming the diagnosis of ALS in a patient with clinical signs that are purely restricted to the UMN. Similarly, magnetic resonance imaging (MRI) evidence of corticospinal tract degeneration or evidence of UMN dysfunction as indicated by ${ }^{1} \mathrm{H}$-magnetic resonance spectro- 
scopy $\left({ }^{1} \mathrm{H}-\mathrm{MRS}\right)$ can be used to demonstrate UMN involvement in a patient with signs restricted to the LMN [2]. Unfortunately, neither technique for the detection of UMN involvement is sensitive since clinical signs usually antedate the appearance of MR abnormalities.

Theories of the etiology of ALS abound, based upon biochemical abnormalities noted in patients or the biological products derived from those patients. Biological markers which have been advanced as potential surrogate markers for the diagnosis of ALS include increased levels of glutamate in the cerebrospinal fluid (CSF) and blood, oxidative products in the blood, abnormal splicing variants of mRNA from EAAT2 (GLT1) in the CSF, DNA repair enzyme defects in cells, and changes of ${ }^{1} \mathrm{H}$-MRS in levels of N-acetyl aspartate (NAA) and glutamate [5].

\section{Use of biological markers in measuring disease progression in therapeutic trials of ALS}

A positive therapeutic trial of an agent effective in ALS should ideally demonstrate a reduced or arrested progression of loss of LMNs and UMNs in patients. There are several excellent techniques for estimating the number of motor units (i.e. LMNs) in specific skeletal muscles. These estimates are relatively reproducible over time in a given patient's muscle, and are quite sensitive to diseaserelated change. Unfortunately the quantitation of UMN loss in ALS is much more difficult. MR studies are relatively insensitive (see above), and cortical magnetic stimulation, which shows abnormalities in ALS [6], does not measure neuronal loss.

Due to these difficulties, we have had to use secondary markers of UMN and LMN loss to measure disease progression in therapeutic trials of ALS. Survival is generally regarded as the 'gold standard' for measuring outcome in ALS and was the basis for demonstrating the efficacy of riluzole. However, although death itself is absolute, it is nevertheless subject to manipulation by various interventions. Bi-level intermittent positive airway pressure (Bi-PAP) support has been shown to increase survival rates [4].

Tracheotomy with permanent assisted ventilation and suicide are opposing influences upon the timing of death and therefore interfere with the reliability of survival as an index of disease progression. Moreover, death occurs at different stages of progression of ALS in different patients, dependent upon the part of the body that was initially and more severely involved.

Muscle strength, timed tests and ALS scales (such as the ALS-Functional Rating Scale) are also used as 'gold standards' for measuring neuromuscular function in clinical therapeutic trials of ALS. There is extensive literature describing these measures and defining their pros and cons. Unfortunately, such tests cannot separate dysfunction due to UMN impairment from that due to LMN; this increases the variability and complexity of interpretation.

The final 'gold standard' of disease progression in ALS is respiratory vital capacity, which also has generated an extensive literature. It is subject to significant variation over time, and like all motor measurements, is affected by both LMN and UMN impairment.

${ }^{1} \mathrm{H}-\mathrm{MRS}$ quantification of motor cortex and brainstem NAA has been shown to decrease with time and progression of ALS. The ability to demonstrate a significant beneficial effect of 3 weeks treatment with riluzole on NAA/ $\mathrm{Cr}$ ratios in the motor cortex of ALS patients has been reported $[5,1,3]$. Statistically significant results were obtained in a study involving 11 riluzole-treated patients and 12 untreated patients [3]. However, we have been unable to substantiate this finding [2].

The major problem for clinical trials in ALS is the biological variability of the disease. Some patients die within a month of onset if the respiratory muscles are the initial sites of affliction, while others may live for decades. One of my patients has had the disease for 37 years, and is still playing golf at the age of 67 years. Different regions of the motor system are involved in different patients, certainly in the earlier stages of the disease. Hence, when any specific parameter is measured in a therapeutic trial, some patients will have no involvement whatsoever throughout the course of the trial, some will have progressive impairment, and some will already have 'bottomed out' even before the trial started. As a consequence of this variability of biological markers for disease progression, in current therapeutic trials very large numbers of patients $(n=250-400)$ are needed for each treatment arm. This is necessary in order to achieve the requisite power to demonstrate a realistic level of therapeutic drug efficacy.

Since such large trials are expensive, it is our duty to try to develop more cost-effective paradigms for therapeutic trials in ALS. There are a number of ways in which this might be achieved. One is to select patients who are in the early but active phase of progression as demonstrated by a certain test paradigm. For instance, a trial could be restricted to patients either showing early loss of motor units in one of the intrinsic hand muscles, or early decrease of vital capacity. Only that measure would be followed in the trial and efficacy would be indicated by a slowed rate of progression of that specific measure. However, it has been believed that regulatory agencies like the Food and Drug Administration would not approve a new drug that demonstrated such a limited effect upon the progression of ALS. Nevertheless, several such demonstrations of efficacy in different sub-groups of patients would probably be acceptable evidence for approval of a new therapeutic agent, and would almost certainly require fewer patients than current large multi-center trials.

An alternative approach is combinations of slopes of deterioration across different tests. In each patient, one or 
more parameters showing evidence of early active progression of the disease could be selected and studied during the course of a double-blinded controlled trial of active treatment versus placebo. Comparison of the combined slopes of different measures would be the basis for determining therapeutic efficacy. This approach does not win the approval of statisticians, who are concerned about the comparison of 'apples and oranges'. Nevertheless, I believe it would be worthwhile researching existing ALS databases to determine if such an approach would have a greater power than the current techniques.

\section{Biological markers in identifying potential therapies for ALS}

Every new theory of the etiology of ALS has given rise to a corresponding novel treatment. In only one instance to date has this proved to be effective, namely the demonstration of the therapeutic efficacy of riluzole based upon the excitotoxic glutamatergic theory. We await with interest the Phase III trial of gabapentin, which is also based on this theory. The use of antioxidant vitamins, derived from observations of oxidative damage in ALS, has unfortunately not proved to be effective in a number of studies. Although deficiency of nerve growth factors does not appear to be responsible for the motor neuron degeneration in ALS, such factors may still prove to be therapeutic. However, so far the subcutaneous administration of several such agents has been ineffective.

In the future, therapeutic drugs should be developed to treat aspects of the degeneration cascade in ALS, for ex- ample, by blocking apoptosis genes and improving axonal transport. Current developments include techniques to block the effect of mutant dominant genes and also to block the effect of genes up-regulated as part of the degeneration cascade. Anti-sense oligonucleotides can be designed to bind to specific mRNA species, preventing translation. Peptide nucleic acids can similarly be designed to bind to specific DNA gene sequences, blocking transcription. These agents might be of value in both familial and sporadic ALS.

Finally, future developments should involve expanding the use of biological markers derived from the exciting field of population pharmacokinetics, based upon DNA chip technology and single nucleotide polymorphisms (SNPs). Such SNPs can provide the DNA 'fingerprint' to identify sub-populations of patients who will respond to a drug, thereby distinguishing them from non-responders.

\section{Conclusions}

Biological markers are essential components in our struggle both to discover the cause of ALS and to find a cure. This is particularly so because of our inability to sample the affected brain and spinal cord tissues in vivo in ALS. However, it is important to appreciate the strengths and weaknesses of biological markers. Our goals must be to refine the biological markers that we currently have, and to develop new ones, since they are essential for diagnosis, clinical trials and the identification of new therapies.

\section{References}

1. Block W, Karitzky J, Traber F, et al (1998) Proton magnetic resonance spectroscopy of the primary motor cortex in patients with motor neuron disease: subgroup analysis and follow-up measurements. Arc Neurol 55:931-936
2. Bowen B, Pattany P, Bradley W (1999)

${ }^{1} \mathrm{H}$ magnetic resonance spectroscopy in ALS. Am J Neuro Radiol (in press)

3. Kalra S, Cashman NR, Genge A, et al (1998) Recovery of N-acetylasparate in corticomotor neurons of patients with ALS after riluzole therapy. Neuroreport 9:1757-1761

4. Kleopas KA, Sherman M, Neal B, et al (1999) Bipap improves survival and rate of pulmonary function decline in patients with ALS. J Neurol Sci 164:82-88
5. Pioro EP, Antel JP, Cashman NR, et al (1994) Detection of cortical neuron loss in motor neuron disease by proton magnetic resonant spectroscopic imaging in vivo. Neurology 44:1933-1938

6. Sommer M, Tergau F, Wischer S, Reimers C-D, Beuche W, Paulus W (1999) Riluzole does not have an acute effect on motor thresholds and the intracortical excitability in amyotrophic lateral sclerosis. J Neurol [Suppl 3] 246: $22-26$ 